\title{
Adipsic diabetes insipidus and SGLT2 inhibitor: A perplexing conundrum
}

\section{Dear Editor,}

A 70-year-old man with poorly controlled type 2 diabetes mellitus (DM), hypertension, hypercholesterolemia and alcohol dependence presented intoxicated, with occipital scalp lacerations after a fall. A brain computerised tomography (CT) revealed occipital skull fracture with bilateral subarachnoid haemorrhages, subdural haemorrhages and parenchymal contusions. He was admitted for close observation in the neurosurgical unit. His regular medications consisted of glipizide, empagliflozin, losartan and simvastatin.

Following admission, he received levetiracetam for seizure prophylaxis, lorazepam and thiamine for delirium tremens, as well as co-amoxiclav and clarithromycin for pneumonia. He did not receive steroids, diuretics or hypertonic saline. Subsequently, as the surgical plan was expectant, he began physical rehabilitation 11 days from admission. Twenty-four days after admission, he developed hypernatremia (serum sodium $155 \mathrm{mmol} / \mathrm{L}$ ), and intravenous (IV) hypotonic dextrose-containing drip was commenced. Despite this, hypernatremia worsened (serum sodium 163-166mmol/L), prompting an endocrine consult on day 30 of admission (Fig. 1).

Preceding hypernatremia, there was polyuria (urine output $3-3.6 \mathrm{~L} /$ day). There was no significant hypercalcaemia nor hypokalemia. Renal function was normal. He did not fulfil the criteria for hyperosmolar hyperglycaemic state, but osmotic diuresis from uncontrolled hyperglycaemia was considered, as capillary blood glucose ranged from $15-15.9 \mathrm{mmol} / \mathrm{L}$ on the day of review. Empagliflozin and glipizide were stopped on day 30, and the patient was initiated on a basal-bolus insulin regime for optimisation of glycaemic control. The patient was observed to prefer cold fluids when offered but was unable to express thirst or request for fluids despite profound clinical dehydration. His blood pressure was $103 / 74 \mathrm{mmHg}$ with a heart rate of 89 beats/min. The volume of IV hypotonic fluid replacement was immediately increased, in addition to scheduled oral fluids. A desmopressin test was conducted with intramuscular desmopressin $2 \mu \mathrm{g}$ on day 30. Before desmopressin administration, hypernatremia (serum sodium $160 \mathrm{mmol} / \mathrm{L}$ ), plasma hyperosmolality $(356 \mathrm{mOsm} / \mathrm{kg})$ and urine osmolality of $529 \mathrm{mOsm} / \mathrm{kg}$ were documented. After administration, urine osmolality increased by $33 \%$ with no corresponding decrease in urine volume.
Following cessation of empagliflozin and improvement in hyperglycaemia, polyuria recurred (Fig. 1). Urine osmolality decreased progressively to $<300 \mathrm{mOsm} /$ $\mathrm{kg}$ despite ongoing hypernatremia (serum sodium 146-157mmol/L) and plasma hyperosmolality (serum osmolality $307-339 \mathrm{mOsm} / \mathrm{kg}$ ). A repeated desmopressin test on day 35 demonstrated a $131 \%$ increase in urine osmolality from $252-582 \mathrm{mOsm} / \mathrm{kg}$ along with significant reduction in urine volume. This was consistent with the diagnosis of complete central diabetes insipidus (DI). Oral desmopressin $100 \mu \mathrm{g}$ nocte was started and increased to $100 \mu \mathrm{g}$ twice daily, with resolution of hypernatremia and polyuria (Fig. 1). Apart from gonadotrophin deficiency of central origin, the rest of the anterior pituitary hormonal profile was normal. Attempted magnetic resonance imaging of the pituitary was unsuccessful despite sedation. Pituitary and hypothalamus CT showed no sellar or suprasellar mass, and near-complete resolution of the cerebral haemorrhages. Despite the improvement in cognitive status, the patient remained persistently adipsic for 6 subsequent weeks. His Glasgow Coma Scale remained at 14-he opened eyes spontaneously (E4), was confused (V4) and obeyed commands (M6). Oral desmopressin $100 \mu \mathrm{g}$ twice daily was continued with scheduled oral fluids 1L/day, strict intake and output charting, daily weight measurements and regular sodium monitoring. He was transferred to a nursing home on discharge with a stable sodium trend.

In polyuria following traumatic brain injury (TBI), the most important diagnosis to consider is DI, a sequela associated with increased mortality. ${ }^{1}$ The hallmark of DI is hypotonic polyuria (urine output $>3 \mathrm{~L} /$ day, urine osmolality $<300 \mathrm{mOsm} / \mathrm{kg}$ ), with concurrent hypernatremia and hyperosmolality if fluid losses are not adequately replaced. In our patient, the initial lack of hypotonic urine may be explained by 2 factors. The first was osmotic diuresis from hyperglycaemia due to poorly controlled DM, which was aggravated by prolonged administration of large volumes of dextrosecontaining drip (paradoxically intended to treat hypernatremia and hyperosmolality). The second important factor was treatment with sodium-glucose cotransporter-2 (SGLT2) inhibitor, which leads to decreased glucose threshold with increased urinary glucose excretion and free water loss. ${ }^{2}$ Indeed, empagliflozin has been reported to lead to severe 


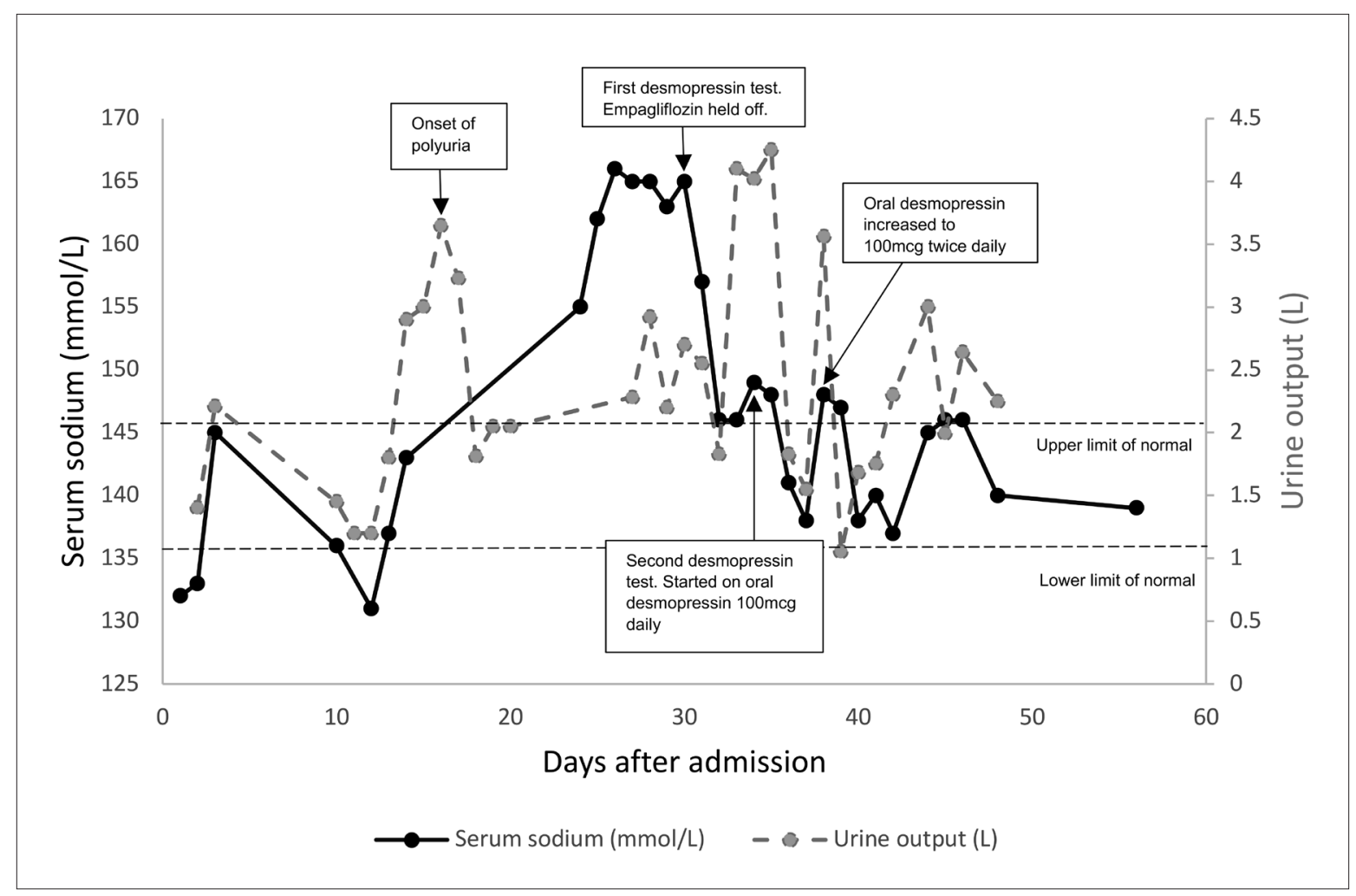

Fig. 1. Trend of serum sodium and urine output throughout entire duration of hospitalisation.

hypernatremic dehydration in a patient unable to drink spontaneously due to pontine stroke. ${ }^{3}$ The development of hypotonic polyuria following improvement in hyperglycaemia and cessation of empagliflozin strengthened our postulation.

Our next step was to determine the aetiology of DI. As direct measurements of vasopressin or copeptin are unavailable in our institution, we relied on the standard indirect water deprivation test to differentiate cranial from nephrogenic DI. In the presence of urine osmolality of $<300 \mathrm{mOsm} / \mathrm{kg}$, an increase in urine osmolality of $\geq 50 \%$, with desmopressin, implied complete central DI, while an increase in urine osmolality of $<10 \%$ implied complete nephrogenic DI. ${ }^{4}$ A maximal urine concentration of $300-$ $800 \mathrm{mOsm} / \mathrm{kg}$ and increase in urine osmolality by $>9 \%$ with desmopressin, as in this patient, suggested partial central or partial nephrogenic DI. ${ }^{4}$ We postulated that the initial partial response to desmopressin was contributed by superimposed nephrogenic DI induced by hyperglycaemia and SGLT2 inhibitor. Evidence of vasopressin resistance has been demonstrated in patients with chronic hyperglycaemia that can be reversed with improved glycaemic control. ${ }^{5}$ Additionally, empagliflozin has been associated with aquaporin downregulation and development of partial nephrogenic DI in pre-clinical studies. ${ }^{6}$ The robust response to a repeated desmopressin test following improvement in hyperglycaemia and cessation of empagliflozin strengthened our postulation.

Although the patient clearly had DI following TBI, there were a few atypical features. While the onset of DI typically occurs 1-9 days following TBI, ${ }^{1}$ in this case the onset of polyuria was delayed at 15 days post-TBI. DI following TBI is usually transient with a median duration of 4 days; ${ }^{1}$ however our patient required desmopressin on discharge and is likely to have permanent DI. A prominent feature in the patient was the persistent inability to sense thirst despite profound hyperosmolality that was out of proportion with the mild cognitive impairment. This was suggestive of adipsic DI, a rare disorder consisting of central DI with a deficient or absent thirst response, and arginine vasopressin release in response to hyperosmolality, which is usually permanent. ${ }^{7}$ The consistent case description confirmed adipsic DI following TBI, which has been described only once in the literature. ${ }^{7}$

There were several other observations worth highlighting. The coexistence of both forms of diabetes is uncommon and has been described in 
craniopharyngioma, pituitary metastases and Wolfram syndrome. The clinical features of Wolfram syndromeoptic atrophy and hearing loss-were absent in this patient. ${ }^{8}$ Hyponatremia was noted on 2 occasions prior to the presentation of DI (Fig. 1). The hyponatremia on day 2 of admission was a result of hyperglycaemia as the corrected serum sodium was normal at $136 \mathrm{mmol} / \mathrm{L}$, although in this patient with a history of alcohol abuse, hypovolemia and beer potomania were also worthy of consideration. On day 12 of admission, hyponatremia (serum sodium $131 \mathrm{mmol} / \mathrm{L}$ ) was likely secondary to hypovolemia since the patient's oral intake was poor and the hyponatraemia responded to intravenous fluids. The triphasic pattern of DI which can occur post- $\mathrm{TBI}^{9}$ was considered, but was unlikely as there was no hypernatremia and/or polyuria from initial DI prior to hyponatremia. Our patient had profound hypernatremia that is linked to increased mortality. In 1 case series with maximum plasma sodium $>160 \mathrm{mmol} / \mathrm{L}$, all cases did not survive. ${ }^{10}$ Furthermore, the patient may have sustained significant diffuse axonal injury to the hypothalamus and pituitary, which may not be detected through CT brain imaging. ${ }^{11}$

It is important to recognise that hyperglycaemia and SGLT2 inhibitor can confound the interpretation of urine osmolality and the indirect water deprivation test, resulting in central DI mimicking nephrogenic DI. Differentiating between central and nephrogenic DI is crucial since therapies differ vastly. The presentation of adipsic DI can be insidious due to the absence of thirstpolyuria and rising serum sodium may provide clues to the onset of DI. Therefore, monitoring of hydration status, urine output and serum sodium is important. Clinical awareness, timely diagnosis and the correct treatment may mitigate the increased mortality associated with DI, a potential complication of TBI. ${ }^{12}$ Lastly, SGLT2 inhibitor should be ceased for patients with TBI since it can confound the evaluation of DI.

\section{REFERENCES}

1. Hannon MJ, Crowley RK, Behan LA, et al. Acute glucocorticoid deficiency and diabetes insipidus are common after acute traumatic brain injury and predict mortality. J Clin Endocrinol Metab 2013;98:3229-37.

2. Masuda T, Muto S, Fukuda K, et al. Osmotic diuresis by SGLT2 inhibition stimulates vasopressin-induced water reabsorption to maintain body fluid volume. Physiol Rep 2020;8:e14360.

3. Gelbenegger G, Buchtele N, Schoergenhofer C, et al. Severe hypernatraemic dehydration and unconsciousness in a caredependent inpatient treated with empagliflozin. Drug Saf Case Rep 2017;4:17.

4. Christ-Crain M, Bichet DG, Fenske WK, et al. Diabetes insipidus. Nat Rev Dis Primers 2019;5:54.

5. McKenna K, Morris AD, Ryan M, et al. Renal resistance to vasopressin in poorly controlled type 1 diabetes mellitus. Am J Physiol Endocrinol Metab 2000;279:E155-60.

6. Chung S, Kim S, Son M, et al. Empagliflozin contributes to polyuria via regulation of sodium transporters and water channels in diabetic rat kidneys. Front Physiol 2019;10:271.

7. Crowley RK, Sherlock M, Agha A, et al. Clinical insights into adipsic diabetes insipidus: a large case series. Clin Endocrinol (Oxf) 2007;66:475-82.

8. Urano F. Wolfram Syndrome: Diagnosis, management, and treatment. Curr Diab Rep 2016;16:6.

9. Tudor RM, Thompson CJ. Posterior pituitary dysfunction following traumatic brain injury: review. Pituitary 2019;22:296-304.

10. Yang YH, Lin JJ, Hsia SH, et al. Central diabetes insipidus in children with acute brain insult. Pediatr Neurol 2011;45:377-80.

11. Figueira Rodrigues Vieira G, Guedes Correa JF. Early computed tomography for acute post-traumatic diffuse axonal injury: a systematic review. Neuroradiology 2020;62:653-60.

12. Tan CL, Alavi SA, Baldeweg SE, et al. The screening and management of pituitary dysfunction following traumatic brain injury in adults: British Neurotrauma Group guidance. J Neurol Neurosurg Psychiatry 2017;88:971-81.

Marvin Chua, ${ }^{* 1} M R C P$, Donovan Yu Kwang Tay, ${ }^{* 1}$ FAMS, Yee Sien $\underline{\mathrm{Ng}},{ }^{1}{ }_{F A M S}, \mathrm{C}$ Rajasoorya, ${ }^{1}{ }_{F R C P}$

${ }^{1}$ Department of General Medicine, Sengkang General Hospital, Singapore

Correspondence: Dr Donovan Yu Kwang Tay, Department of General Medicine, Sengkang General Hospital, 110 Sengkang East Way, Singapore 544886.

Email: donovan.tay.y.k@singhealth.com.sg

* Joint first authors 